Mathematical Models of Hysteresis 


\author{
I.D. Mayergoyz
}

\title{
Mathematical Models of Hysteresis
}

With 167 Illustrations

Springer-Verlag

New York Berlin Heidelberg London Paris Tokyo Hong Kong Barcelona 
I.D. Mayergoyz

Electrical Engineering Department

Institute for Advanced Computer Studies

University of Maryland

College Park, Maryland 20742

Library of Congress Cataloging-in-Publication Data

Mayergoyz, I.D.

Mathematical models of hysteresis / I.D. Mayergoyz.

p. $\mathrm{cm}$.

Includes index.

ISBN-13:978-1-4612-7767-5

1. Hysteresis-Mathematical models. I. Title.

QC754.2.H9M38 1991

$538^{\prime} .3-\mathrm{dc} 20$

90-39253

CIP

Printed on acid-free paper

(C) 1991 Springer-Verlag New York Inc.

Softcover reprint of the hardcover 1st edition 1991

All rights reserved. This work may not be translated or copied in whole or in part without the written permission of the publisher (Springer-Verlag New York, Inc., 175 Fifth Avenue, New York, NY 10010, USA), except for brief excerpts in connection with reviews or scholarly analysis. Use in connection with any form of information storage and retrieval, electronic adaptation, computer software, or by similar or dissimilar methodology now known or hereafter developed is forbidden.

The use of general descriptive names, trade names, trademarks, etc., in this publication, even if the former are not especially identified, is not to be taken as a sign that such names, as understood by the Trade Marks and Merchandise Marks Act, may accordingly be used freely by anyone.

Typeset by Asco Trade Typesetting Ltd., Quarry Bay, Hong Kong.

$\begin{array}{lllllllll}9 & 8 & 7 & 6 & 5 & 4 & 3 & 2 & 1\end{array}$

ISBN-13:978-1-4612-7767-5

e-ISBN-13:978-1-4612-3028-1

DOI: $10.1007 / 978-1-4612-3028-1$ 
To my children

Sasha and Anna 


\section{Preface}

The purpose of this book is to describe in sufficient detail the mathematical models of hysteresis nonlinearities with "nonlocal memories." The distinct feature of these nonlinearities is that their future states depend on past histories of input variations. It turns out that memories of hysteresis nonlinearities are quite selective. Indeed, experiments show that only some past input extrema (not the entire input variations) leave their marks upon future states of hysteresis nonlinearities. Thus, special mathematical tools are needed to describe nonlocal selective memories of hysteresis nonlinearities. The origin of such tools can be traced back to the landmark paper of Preisach.

The book is primarily concerned with Preisach-type models of hysteresis. All these models have a common generic feature; they are constructed as superpositions of simplest hysteresis nonlinearities-rectangular loops. The discussion is by and large centered around the following topics: various generalizations and extensions of the classical Preisach model (with special emphasis on vector generalizations), finding of necessary and sufficient conditions for the representation of actual hysteresis nonlinearities by various Preisach-type models, solution of identification problems for these models, and numerical implementation and experimental testing of Preisach-type models. Although the study of Preisach-type models constitutes the main subject of the book, some effort is also made to establish some interesting connections between these models and such topics as the critical state model for superconducting hysteresis, the classical Stoner-Wohlfarth model for vector magnetic hysteresis, thermal activationtype models for viscosity, magnetostrictive hysteresis and neural networks.

To make the book accessible to a broader audience, the exposition of the material is purposely made somewhat wordy with the hope that this will contribute to the clarity. This is especially true for the first chapter, while the discussion in the second and third chapters is more concise. The mathematical tools used in the book are quite simple. The exception is the discussion of the identification problems for the vector Preisach models in the third chapter, where some machinery of integral equations and the theory of irreducible representations of the group of rotations is occasionally used. No attempt is made to refer to all relevant publications. For this reason, the reference list is not exhaustive but rather suggestive; the presentation of the material in the book is 
largely based on my publications which are given at the end of the reference list. I first heard about the Preisach model during my conversation with Professor K.M. Polivanov. This was about seventeen years ago, and at that time I lived in Russia. Shortly thereafter, my interest in the Preisach model was strongly enhanced as a result of brief discussions I had with Professor M.A. Krasnoselskii and his colleague A. Pokrovskii. When I came to the United States, my work on hysteresis modelling was strongly encouraged and supported by Dr. O. Manley from the U.S. Department of Energy. My research on the Preisach models has also strongly benefitted from many penetrating discussions I have had with Professor D. Fredkin (University of California, San Diego) over the past five years. I have also been fortunate to have such wonderful graduate students as T. Doong, G. Friedman, C. Korman, and A. Adly, who have assisted me at different times in my work on hysteresis. I am very thankful to Mrs. P. Keehn who patiently and diligently typed several versions of the manuscript. Finally, I gratefully acknowledge the financial support for my research on hysteresis from the U.S. Department of Energy, Engineering Research Program. 


\section{Contents}

Preface $\ldots \ldots \ldots \ldots \ldots \ldots \ldots \ldots \ldots \ldots \ldots \ldots \ldots \ldots$, vii

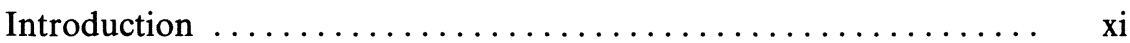

Chapter I. The Classical Preisach Model of Hysteresis .......... 1

1 Definition of the Classical Preisach Model ................. 1

2 Geometric Interpretation and Main Properties of the Preisach Model ............................... 7

3 Experimental Determination of $\mu(\alpha, \beta)$. Representation Theorem .. 18

4 Numerical Implementation of the Preisach Model .......... 32

5 The Preisach Model and Hysteretic Energy Losses ........... 44

6 Preisach Model with Stochastic Input as a Model for Viscosity ... 55

Chapter II. Generalized Scalar Preisach Models of Hysteresis ...... 64

1 Moving Preisach Model of Hysteresis . ................. 64

2 Nonlinear Preisach Model of Hysteresis . . . . . . . . . . . . . . 70

3 Restricted Preisach Models of Hysteresis . . . . . . . . . . . . . 87

4 Dynamic Preisach Models of Hysteresis . . . . . . . . . . . . . 99

5 Preisach Model of Hysteresis with Accommodation ............. 108

6 Superconducting Hysteresis and Preisach-Type Models ........ 114

7 Magnetostrictive Hysteresis and Preisach Models with Two Inputs 122

8 Experimental Testing of Preisach-Type Models of Hysteresis . . . . 129

Chapter III. Vector Presiach Models of Hysteresis . . . . . . . . . 141

1 Classical Stoner-Wohlfarth Model of Vector Hysteresis ........ 141

2 Definition of Vector Preisach Models of Hysteresis and Their Numerical Implementation ......................... 149

3 Some Basic Properties of Vector Preisach Hysteresis Models .... 157

4 Identification Problem for Isotropic Vector Preisach Models ..... 172

5 Identification Problem for Anisotropic Vector Preisach Models ... 179

6 Dynamic Vector Preisach Models of Hysteresis . . . . . . . . . . . 188

7 Generalized Vector Preisach Models of Hysteresis . . . . . . . . 194

References .................................... 202

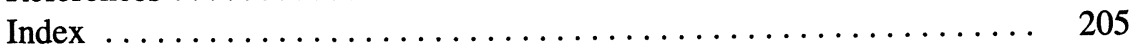




\section{Introduction}

The topic being discussed in this book is mathematical models of hysteresis. Special emphasis is placed on the mathematical exposition of these models which makes them quite general and applicable to the description of hysteresis of different physical nature. There are, however, two additional reasons for this emphasis. As was pointed out by A. Einstein [1], "mathematics enjoys special esteem, above all other sciences [because] its laws are absolutely certain and indisputable ...." Mathematics has achieved and maintained this exceptional position because its results are derived from a few (more or less self-evident) axioms by a chain of flawless reasonings. Since it is based on impeccable logic, mathematics can provide some level of security (and clarity) for natural sciences which is not attainable otherwise. For this reason, the rigorous mathematical representation of natural sciences is highly dcsirable and should be attempted whenever it is possible. In addition, mathematics more and more often serves as a vehicle of communication between scientists and engineers of different specializations. As a result, if some area of science is represented in a rigorous mathematical form, its accessibility is strongly enhanced. With these thoughts in mind, it is hoped that the mathematical exposition of hysteresis models undertaken here will bring much needed clarity into this area and will make it appealing to the broader audience of inquiring researchers.

This monograph has been written by an engineer for engineers. For this reason, mathematics is largely used here as a tool for achieving clarity. As a result, many mathematical subtleties of hysteresis modelling are omitted. These subtleties are by and large related to the fact that hysteresis operators are naturally defined on sets of piecewise monotonic functions which do not form complete function spaces. This leads to the problem of continuous extension of hysteresis operators from the above sets to some complete function spaces. The reader interested in this type of mathematical problems is referred to the study by the Russian mathematicians M. Krosnoselskii and A. Pokrovskii [2].

The phenomenon of hysteresis has been with us for ages and has been attracting the attention of many investigators for a long time. The reason is 
that hysteresis is ubiquitous. It is encountered in many different areas of science. Examples include magnetic hysteresis, ferroelectric hysteresis, mechanical hysteresis, superconducting hysteresis, adsorption hysteresis, optical hysteresis, electron beam hysteresis, etc. However, the very meaning of hysteresis varies from one area to another, from paper to paper, and from author to author. As a result, a stringent mathematical definition of hysteresis is needed in order to avoid confusion and ambiguity. Such a definition will serve a twofold purpose: first, it will be a substitute for vague notions, and, second, it will pave the road for more or less rigorous proofs.

We begin with the definition of scalar hysteresis and, for the sake of generality, we adopt the language of control theory. Consider a transducer (see Fig. 1) which can be characterized by an input $u(t)$ and an output $f(t)$. This transducer is called a hysteresis transducer $(H T)$ if its input-output relationship is a multibranch nonlinearity for which branch-to-branch transitions occur after input extrema. This multibranch nonlinearity is shown in Fig. 2. For the most part, the case of static hysteresis nonlinearity will be discussed. The term "static" means that branches of such hysteresis nonlinearities are determined only by the past extremum values of input, while the speed (or particular manner) of input variations between extremum points has no influence on branching. This statement is illustrated by Figs. 3a, 3b, and $3 \mathrm{c}$. Figures $3 \mathrm{a}$ and $3 \mathrm{~b}$ show two different inputs $u_{1}(t)$ and $u_{2}(t)$ which successively assume the same extremum values but vary differently between these values. Then, for a static $H T$, these two inputs will result in the same $f-u$

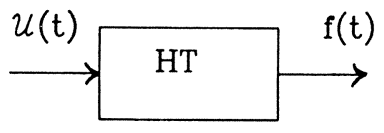

Fig. 1

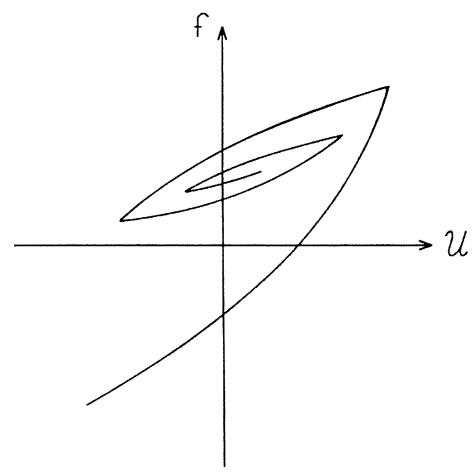

Fig. 2 

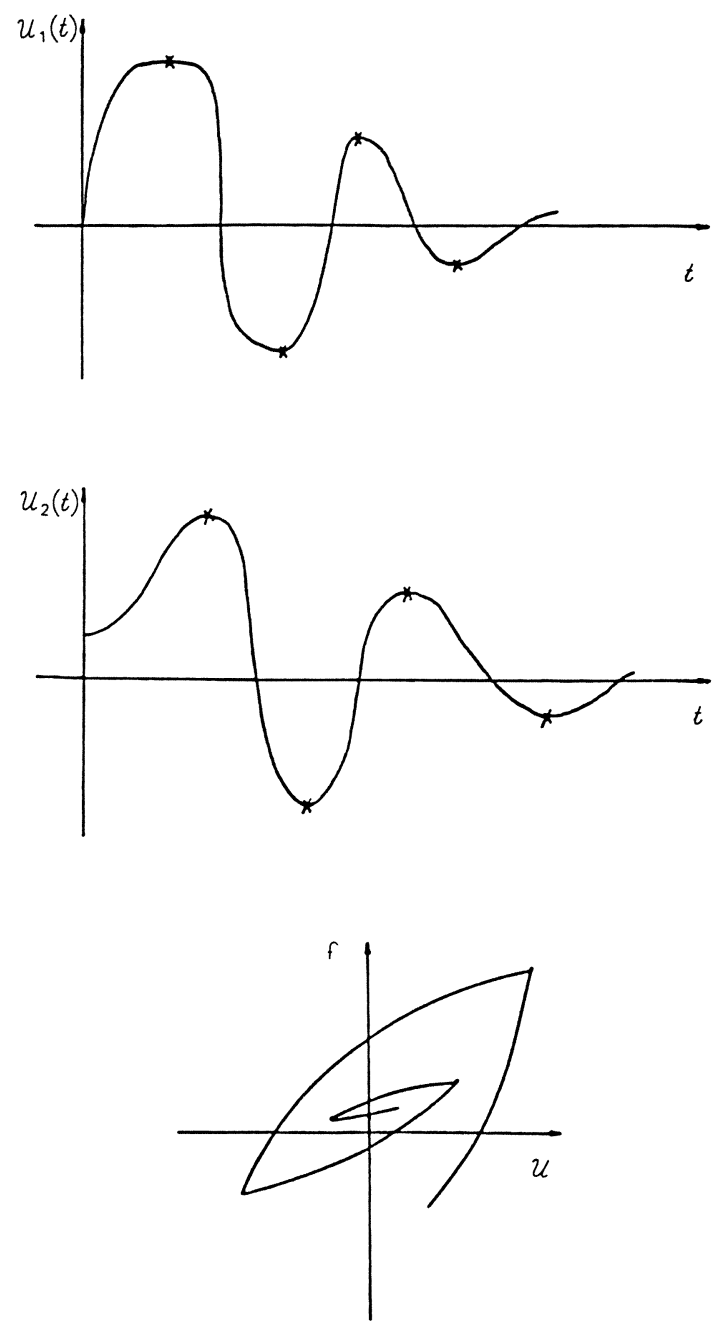

Fig. 3

diagram (see Fig. 3c), provided that the initial state of the transducer is the same for both inputs.

The given definition of static scalar hysteresis is consistent with existing experimental facts. Indeed, it is known in the area of magnetic hysteresis that a shape of major (or minor) loop (see Fig. 4) can be specified without referring to how fast magnetic field $H$ varies between two extremum values, $+H_{m}$ and $-H_{m}$. This indicates that time effects are negligible and the given definition of a static hysteresis transducer is an adequate one. It is worthwhile to keep in mind that, for very fast input variations, time effects become important and 


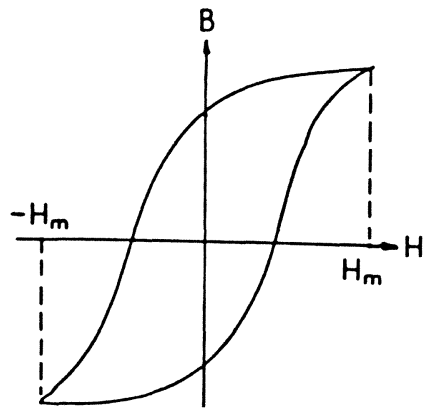

Fig. 4

the given definition of static hysteresis fails. In other words, this definition (as any other definition) has its limits of applicability to real life problems.

In the existing literature, the hysteresis phenomenon is by and large linked with the formation of hysteresis loops (looping). This may be misleading and create the impression that looping is the essence of hysteresis. In this respect, the given definition of hysteresis emphasizes the fact that branching constitutes the essence of hysteresis, while looping is a particular case of branching. Indeed, looping occurs when the input varies back and forth between two consecutive extremum values, while branching takes place for arbitrary input variations.

From the given definition, it can also be concluded that scalar hysteresis can be interpreted as a nonlinearity with a memory which reveals itself through branching.

In the given definition of hysteresis, the physical meanings of the input $u(t)$ and the output $f(t)$ were left unspecified. This was done deliberately, for the sake of mathematical generality. However, it is not difficult to specify the meanings of $u(t)$ and $f(t)$ in particular applications. For instance, in magnetism $u(t)$ is the magnetic field and $f(t)$ is the magnetization, in mechanics $u(t)$ is the force and $f(t)$ is the displacement (length), in adsorption $u(t)$ is the gas pressure and $f(t)$ is the amount of material adsorbed. The notion of a hysteresis transducer may have different interpretations as well. For instance, in magnetism the $H T$ can be construed as an infinitesimally small volume of magnetic material, and the corresponding input-output hysteresis nonlinearity can be interpreted as a constitutive equation for this material.

All static hysteresis nonlinearities fall into two general classifications: (a) hysteresis nonlinearties with local memories and (b) hysteresis nonlinearities with nonlocal memories. The hysteresis nonlinearities with local memories are characterized by the following property. The value of output $f\left(t_{0}\right)$ at some instant of time $t_{0}$ and the values of input $u(t)$ at all subsequent instants of time $t \geq t_{0}$ uniquely predetermine the value of output $f(t)$ for all $t>t_{0}$. In other words, for hysteresis transducers with local memories the past exerts its influence upon the future through the current value of output. This is not the 


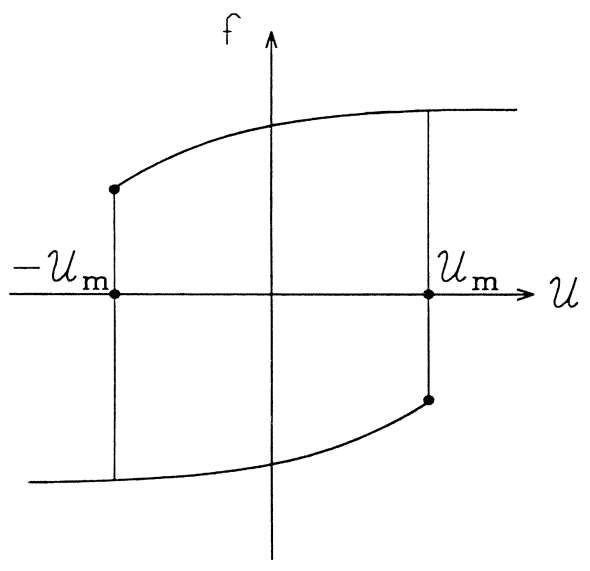

Fig. 5

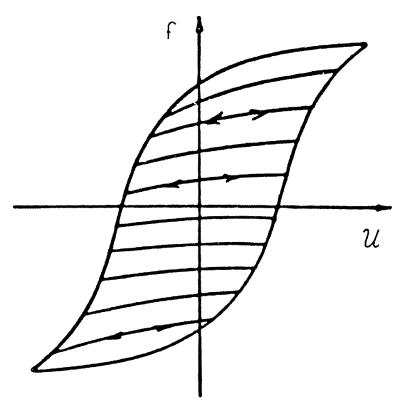

Fig. 6

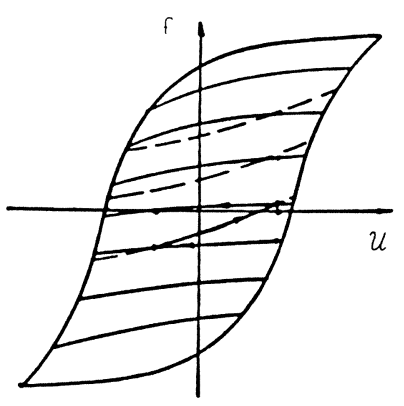

Fig. 7

case for hysteresis transducers with nonlocal memories. For such transducers, future values of output $f(t),\left(t \geq t_{0}\right)$ depend not only on the current value of output $f\left(t_{0}\right)$ but on past extremum values of input as well.

The typical examples of hysteresis nonlinearities with local memories are shown in Figs. 5, 6, and 7. Figure 5 shows the simplest hysteresis nonlinearity with local memory. It is specified by a major loop which is formed by ascending and descending branches. These branches are only partially reversible (their vertical sections are not reversible). This type of hysteresis nonlinearity is characteristic, for instance, for single Stoner-Wohlfarth magnetic particles [3]. For this type of hysteresis, branching occurs if only extremum values of input exceed $+u_{m}$ or $-u_{m}$.

A more complicated type of hysteresis nonlinearities with local memories is illustrated by Fig. 6 . Here, there is a set of inner curves within the major loop and only one curve passes through each point in the $f-u$ diagram. These curves are fully reversible and can be traversed in both directions, for a 
monotonically increasing and decreasing input $u(t)$. For this type of hysteresis, branching may occur only when ascending or descending branches of major loops are reached.

A hysteresis nonlinearity with local memory which has two sets of inner curves (the ascending and descending curves) is shown in Fig. 7. This type of hysteresis was probably first described by Madelung [4] at the beginning of the century, and afterwards it was independently invented by many authors time and time again (see, for instance, [5] and [6]). For this hysteresis nonlinearity, only one curve of each set passes through each point in the $f-u$ diagram. If the input $u(t)$ is increased, the ascending curve is followed, if it is decreased, the descending curve is traced. Thus, branching occurs for any input extremum. However, in general, minor loops are not formed; if $u(t)$ varies back and forth between the same two values, the output usually exhibits a continued upward drift (see Fig. 7).

It is clear from the above examples that all hysteresis nonlinearities with local memories have the following common feature: every reachable point in the $f-u$ diagram corresponds to a uniquely defined state. This state predetermines the behavior of $H T$ in exactly one way for increasing $u(t)$ and exactly one way for decreasing $u(t)$. In other words, at any point in the $f-u$ diagram there are only one or two curves that may represent the future behavior of $H T$ with local memory (see Fig. 8). This is not true for hysteresis transducers with nonlocal memories. In the latter case, at any reachable point in the $f-u$ diagram there is an infinity of curves that may represent the future behavior of the transducer (see Fig. 9). Each of these curves depends on a particular past history, namely, on a particular sequence of past extremum values of input. By analogy with the random process theory, hysteresis nonlinearities with local memories can be called markovian hysteresis nonlinearities, while hysteresis nonlinearities with nonlocal memories are nonmarkovian. It is clear that hysteresis nonlinearities with nonlocal memories are much more complicated than those with local memories.

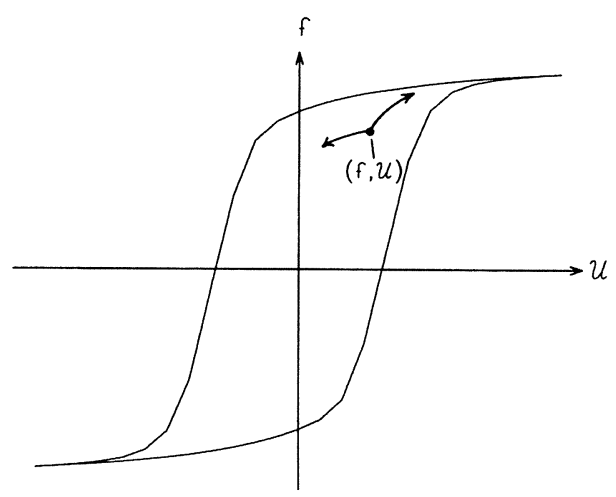

Fig. 8 


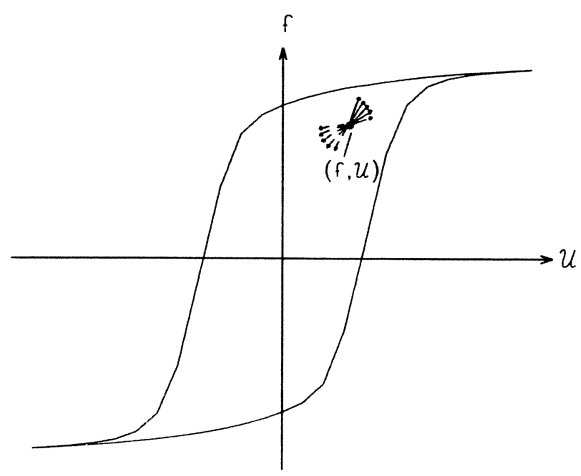

Fig. 9

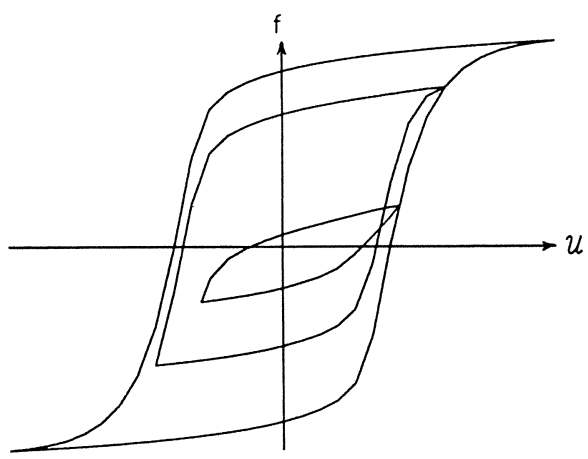

Fig. 10

Mathematical models of hysteresis nonlinearities with local memories have been extensively studied by using differential and algebraic equations. These models have achieved a high level of sophistication which is reflected, for instance, in publications [7-10]. However, the notion of hysteresis nonlinearities with local memories is not consistent with experimental facts. For instance, it is reported in [21] that crossing and partially coincident minor loops have been experimentally observed. These loops are schematically shown in Fig. 10 and 11, respectively. The existence of crossing minor loops attached to a major loop is more or less obvious, while the presence of partially coincident minor loops is a more subtle phenomenon. The existence of crossing and partially coincident minor loops clearly suggests that the states of the corresponding hysteresis transducers are not uniquely specified by their inputs and outputs. Thus, hysteresis of this transducer does not have a local memory.

This book is solely concerned with mathematical models of hysteresis with nonlocal memory. The question arises, why are these models needed? The answer is that the hysteresis transducer is usually a part of a system. As a 


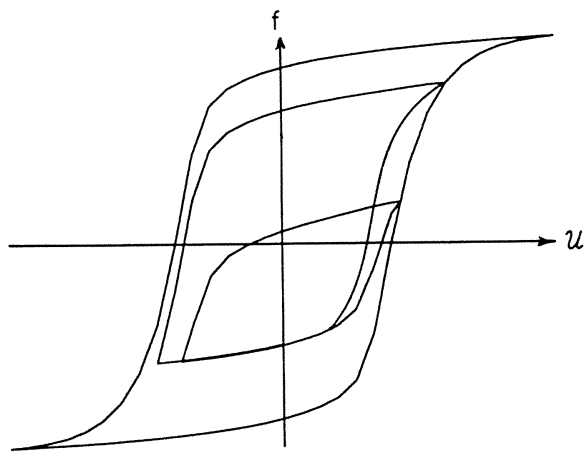

Fig. 11

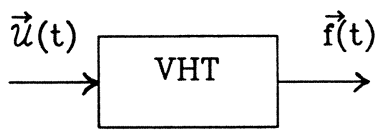

Fig. 12

result, its input is not known beforehand, but is determined by the interaction of the transducer with the rest of the system. Since the input of HT is not predictable a priori, it is impossible to specify ahead of time the branches of hysteresis nonlinearity which will be followed in a particular regime of the system. This is the main impediment as far as self-consistent mathematical descriptions of systems with hysteresis are concerned. To overcome the difficulty mentioned above, mathematical models of hysteresis are needed. These models represent new mathematical tools which themselves (due to their structure) will detect and accumulate input extrema and will choose appropriate branches of the hysteresis nonlinearity with respect to the accumulated histories. Coupled together with mathematical description of the rest of the system these models will constitute complete and self-consistent mathematical descriptions of systems with hysteresis. Without such models, the self-consistent mathematical descriptions of systems with hysteresis are virtually impossible.

We next turn to the discussion of vector hysteresis. This hysteresis can be characterized by a vector input $\vec{u}(t)$ and vector output $\vec{f}(t)$ (see Fig. 12). Two- and three-dimensional vector inputs and vector outputs are most relevant to practical applications. That is why only two- and three-dimensional vector hysteresis models are discussed in this book. However, the formal mathematical generalization of these models to $n$ dimensions $(n>3)$ is straightforward. It is believed that such a generalization will be performed by the reader if needed.

The most immediate problem we face is how to define vector hysteresis in a mathematically rigorous as well as physically meaningful way. To do this, 
it is important to understand what constitutes the essential part of past input history in the case of vector hysteresis. In other words, we need to understand what part of the past input history affects the future values of output. To resolve this problem, it would be natural to turn to experimental facts. Unfortunately, the experimental data available in the area of vector hysteresis are quite scarce and inconclusive. The latter means that these data provide no clear indications as to how the problem posed above can be resolved. Nevertheless, it is possible to find some resolution of this problem by strictly logical reasoning. The starting point for our inference is the notion that scalar hysteresis is a particular case of vector hysteresis. This case is realized when the vector input $\vec{u}(t)$ is restricted to vary along only one direction. In fact, it can be successfully argued (at least in the area of magnetics) that there is no such a thing as scalar hysteresis. Whenever we talk about scalar hysteresis, we are actually dealing with some specific properties of vector hysteresis which have been observed for input variations along some fixed directions. Thus, it can be concluded that vector hysteresis is reduced to scalar hysteresis when the vector input is restricted to vary along an arbitrarily fixed direction. Since the above reduction occurs for any fixed direction and since past extremum values of input constitute the essential part of past history for scalar hysteresis, it is natural to conclude that in the case of vector hysteresis past extremum values of input projections along all possible directions may leave their mark upon the future. As a result, we arrive at the following definition of vector hysteresis. Vector hysteresis is a vector nonlinearity with the property that past extremum values of input projections along all possible directions may affect future values of output. Since no mention is made concerning the speed of input variations, it is apparent that the above definition implies static vector hysteresis. It is also clear that mathematical models of vector hysteresis are imperative for self-consistent descriptions of systems with vector hysteresis. These models should be able to detect and store past extrema of input projections along all possible directions and choose the appropriate value of vector output according to the accumulated history.

This book deals exclusively with the mathematical models of hysteresis which are purely phenomenological in nature. Essentially, these models represent the attempt to describe and generalize experimental facts. They provide no insights into specific physical causes of hysteresis. Nevertheless, they have been and may well continue to be powerful tools for device design. There are, however, fundamental models of hysteresis which attempt to explain experimental facts from first principles. For instance, in micromagnetics, these principles require that the equilibrium distribution of magnetization should correspond to an energy minimum. The minimized energy basically includes the exchange energy, the anisotropy energy, the energy of interaction with an applied field, the magnetostatic self-energy, and possibly some other terms. It turns out that there are many (at least two) different local minima of the total energy for a given applied field. Since only one of these energy minima corresponds to the thermodynamic equilibrium state, the others must be 
metastable. They may persist for very long times. These pcrsisting metastable states are responsible for the origin of hysteresis.

Although the above micromagnetic approach is fundamental in nature, its implementation encounters some intrinsic difficulties.

First, in order to carry out this approach, the detailed information of microscopic material structure is needed. Only on the basis of this information, can the above-mentioned terms of minimized energy be specified. However, the detailed knowledge of material microstructure is often not available.

Second, the micromagnetic approach leads to nonlinear differential (or integrodifferential) equations which are quite complicated to solve even using sophisticated numerical techniques. In part, this is because the solution of these equations may exhibit highly irregular behavior. Indeed, domains and their walls should emerge from the micromagnetic approach. The domain walls are small regions where the direction of magnetization changes quite rapidly, from some particular direction in one domain to a different direction in an adjacent domain. In a way, these domain walls can be mathematically construed as interior layers. This suggests that micromagnetic problems may well belong to the class of singularly perturbed problems. (This fact has not been appreciated enough in the existing literature.) To resolve the fast variations of magnetization over the domain walls, very fine meshes are needed. But, the domain walls usually move when the applied field is changed. Thus, it is not clear a priori where the fine meshes should be located. This may seriously complicate the numerical analysis.

Finally, the detailed domain structure which can be produced by the micromagnetic approach may be irrelevant to some practical problems. This is the case, for instance, in the design of devices for which the average value of magnetization over regions with dimensions much larger than domain dimensions is of interest.

Summarizing the above discussion, it can be concluded that the phenomenological approach is more directly connected with macroscopic experimental data. For this reason, it is of a great value to the device design. The fundamental micromagnetic approach, on the other hand, is intimately related to material structure and, therefore, it can be useful in the design of new materials. 